NASA Contractor Report 4247

\title{
A Chebyshev Matrix Method for Spatial Modes of the Orr-Sommerfeld Equation
}

G. Danabasoglu and S. Biringen

University of Colorado

Boulder, Colorado

Prepared for

Langley Research Center

under Grant NAG1-462

\section{N/SA}

National Aeronautics and

Space Administration

Office of Management

Scientific and Technical

Information Division

\section{9}




\title{
A Chebyshev Matrix Method for Spatial Modes of the Orr-Sommerfeld Equation
}

\author{
G. Danabasoglu and S. Biringen \\ Department of Aerospace Engineering Sciences \\ University of Colorado \\ Boulder, Colorado 80309
}

The Chebyshev matrix collocation method is applied to obtain the spatial modes of the Orr-Sommerfeld equation for Poiseuille flow and the Blausius boundary layer. The problem is linearized by the companion matrix technique for semi-infinite domain using a mapping transformation. The method can be easily adapted to problems with different boundary conditions requiring different transformations.

\section{INTRODUCTION}

The linear stability of hydrodynamic problems is governed by the Orr-Sommerfeld equation. For parallel shear flows, along with homogeneous boundary conditions, this equation constitutes an eigenvalue problem. For temporally evolving flows, the eigenvalue is the complex frequency and the problem becomes linear in the eigenvalue. Although for small amplitude disturbances temporal evolution is a good approximation to the 
laboratory flow, the correct physics of the problem can be obtained only through the calculation of spatially evolving modes. In the Orr-Sommerfeld equation, these modes are given in terms of the wave number and for these situations, where the complex wave number is the eigenvalue, the Orr-Sommerfeld equation becomes a nonlinear eigenvalue problem.

The numerical solution of the Orr-Sommerfeld equation by matrix methods requires an efficient means of discretization. Since shear flows have steep gradients near the boundaries, it is natural to seek for a discretization with clustering or streching in these regions. Orszag' 's pioneering work demonstrated the usefulness and accuracy of Chebyshev spectral methods for solving the linear eigenvalue problems. Chebyshev methods naturally cluster collocation points in the vicinity of the boundaries and are more accurate than fourth-order finite differences on a stretched mesh with the same number of grid points.

Several techniques to solve the temporal (linear) eigenvalue problem are discussed by Canuto et al. ${ }^{2}$, where the Chebyshev-tau method is favored over the Chebshev collocation matrix method because of the difficulties in the latter concerning the imposition of the boundary conditions. However, the collocation matrix method is easier to formulate and the boundary conditions do not pose a problem provided that they are applied near the boundaries, i. e. only the first and last few rows of the matrix are modified. Furthermore, the Chebyshev-tau method requires major modifications for each new mean velocity profile or for a new coordinate transformation ${ }^{3}$. Under similar conditions, the Chebyshev collocation matrix method necessitates no significant changes. Recently, Laurien ${ }^{4}$ implemented the Chebyshev matrix collocation method to solve the temporal generalized eigenvalue problem for the Blausius boundary layer and obtained very good results. Laurien ${ }^{4}$ used an even number of collocation points obviating the need for the explicit imposition of the homogeneous boundary conditions at infinity. Note that Chebyshev polynomials automatically satisfy these conditions at the mid-point of the Chebyshev collocation domain which corresponds to infinity in the physical space. Using his tech- 
nique with an odd number of collocation points, Biringen and Danabasoglu ${ }^{5}$ included all the boundary conditions and still obtained results in excellent agreement with literature.

The solution of nonlinear eigenvalue problems can be obtained efficiently by shooting methods in which a good initial guess is vital for convergence. For problems in which a good initial guess is not available, the matrix method presents an attractive alternative. Bridges and Morris ${ }^{6}$ developed a companion matrix method to linearize the spatial eigenvalue problem to study the stability of both channel and boundary layer flows. Their method incorporates the Chebyshev-tau method to discretize the governing equation. Once the problem is linearized by this method, the entire spectrum can be obtained via the QZ algorithm. Recently, Khorrami et al. ${ }^{3}$ used the Chebyshev collocation matrix method to study the temporal and spatial stability of swirling flows in enclosed domains. They employed the companion matrix method of Bridges and $M o r r i s^{6}$, however they did not present the eigenvector distributions corresponding to the least damped eigenvalue.

In this work, the Chebyshev collocation matrix method is combined with the companion matrix method to solve the non-linear spatial eigenvalue problem for channel and Blasius boundary layer flows. The semi-infinite domain of the Blausius flow requires a mapping transformation and presents a challenging problem for the method under consideration. In addition to the least damped eigenvalues, we also present the corresponding eigenfunction distributions.

\section{GOVERNING EQUATIONS}

The stability of the parallel shear flows is governed by the Orr-Sommmerfeld equation,

$$
\left[\left(\frac{d^{2}}{d y^{2}}-\alpha^{2}\right)^{2}-i \operatorname{Re}\left\{(\alpha U-\omega)\left(\frac{d^{2}}{d y^{2}}-\alpha\right)-\alpha U^{\prime \prime}\right\}\right] \phi=0
$$

with

$$
\begin{gathered}
\phi=\phi^{\prime}=0 \text { at } y= \pm 1, \\
\phi=\phi^{\prime}=0 \text { at } y=0, y \rightarrow \infty .
\end{gathered}
$$


Equations (1a) and (1b) constitude the boundary conditions for channel and boundary layer flows, respectively. For spatial modes, $\alpha$ is the complex wave number and $\omega$ is the real, radian frequency. Also $U$ and $U^{\prime \prime}$ represent the base flow velocity profile and its second derivative, respectively. $R e$ is the Reynolds number defined as $R e=U_{0} \delta_{1} / \nu$ for the boundary layer and $R e=U_{1} h / \nu$ for channel flow. Here, $\nu$ is the kinematic viscosity of the fluid, $h$ is the half channel height and $\delta_{1}$ is the displacement thickness. Velocity components are non-dimensionalized by the free stream velocity $\left(U_{0}\right)$ and the maximum channel velocity $\left(U_{1}\right)$ for the channel and boundary layer cases, respectively.

Note that in the derivation of equation (1), the two-dimensional normal mode expansion is applied using the stream function $(\psi)$ formulation. Accordingly,

$$
\psi=\phi(y) e^{i(\alpha x-\omega t)}
$$

This expansion gives the following equations for the horizontal and vertical perturbation velocities, respectively,

$$
\begin{gathered}
u=\phi^{\prime}(y) e^{i(\alpha x-\omega t)}, \\
v=-i \alpha \phi(y) e^{i(\alpha x-\omega t)} .
\end{gathered}
$$

\section{SOLUTION PROCEDURE}

The applied numerical procedure uses the Chebyshev-Gauss-Lobatto points for the $\mathrm{y}$-direction discretization

$$
\eta_{j}=\cos (\pi j / N), \quad j=0,1, \ldots N
$$

where $N$ is the number of intervals in the domain and $\eta$ represents the coordinates of the collocation points in the Chebyshev domain. Recall that the Chebyshev polynomials of order $k$ are defined on the interval $(-1,1)$ by

$$
T_{k}(\eta)=\cos \left(k \cos ^{-1} \eta\right)
$$


and $\phi(\eta)$ is expanded in Chebyshev polynomials

$$
\phi(\eta)=\sum_{k=0}^{N} a_{k} T_{k}(\eta)
$$

where $a_{k}$ 's are the expansion coefficients.

The solution of equation (12) depends on the basic state velocity profiles. For channel flow, the velocity profiles are readily given as

$$
U(y)=1-y^{2}, \quad V=0 .
$$

Note that channel boundaries are located at $y= \pm 1$ and they coincide with the domain of the Chebysher polynomials, i. e. $y=\eta$.

The calculation of the basic state velocity profile for the boundary layer is more involved and requires the solution of the Blausius boundary layer equation,

$$
f^{\prime \prime \prime}+\frac{f f^{\prime \prime}}{2}=0
$$

with

$$
\begin{aligned}
f(0) & =0, \\
f^{\prime}(0) & =0, \\
f^{\prime \prime}(0) & =0.332057339 .
\end{aligned}
$$

Then,

$$
U(y)=f^{\prime}(y)
$$

Note that the free stream velocity is assumed to be unity. The solution of equation (9) is obtained by a point-by-point Runge-Kutta-Verner method.

The boundary layer calculations require the mapping of the physical domain onto the Chebyshev domain. In this work, we employ an exponential transformation given by Laurien $^{3}$ which maps the domain from $[0, \infty]$ to $[1,0]$,

$$
\eta=e^{-y / Y}
$$


Here, $Y(Y=20)$ is a mesh clustering parameter, and the boundary points are included in the domain satisfying the boundary conditions exactly.

It is now convenient to write equation (1) in a different form,

$$
\left[\left(\mathbf{D}^{2}-\alpha^{2} \mathbf{I}\right)^{2}-i \operatorname{Re}\left\{(\alpha U \mathbf{I}-\omega \mathbf{I})\left(\mathbf{D}^{2}-\alpha \mathbf{I}\right)-\alpha U^{\prime \prime} \mathbf{I}\right\}\right] \phi=0
$$

where $\mathbf{D}$ represents the Chebyshev collocation matrix which gives the first derivative in the Chebyshev domain. The elements of $D$ can be written as ${ }^{1}$

$$
\begin{aligned}
& \mathbf{D}_{k j}=\frac{c_{k}}{c_{j}} \frac{(-1)^{k+j}}{\eta_{k}-\eta_{j}} \quad(k \neq j), \\
& \mathbf{D}_{j j}=-\frac{\eta_{j}}{2\left(1-\eta_{j}^{2}\right)}, \\
& \mathbf{D}_{00}=-\frac{2 N^{2}+1}{6}=-\mathbf{D}_{N N},
\end{aligned}
$$

and

$$
j, k=0,1, \ldots N
$$

The higher order derivatives are simply obtained as powers of $\mathbf{D}$, i. e.

$$
\mathrm{D}_{p}=\mathrm{D}^{p}
$$

where $p$ is the order of the derivative. In equation (12), $\mathbf{I}$ is the identity matrix.

Note that equation (12) is a polynomial in $\alpha$ with matrix coefficients and has the following explicit form,

$$
\mathbf{R}_{4}(\alpha)=\mathbf{C}_{4} \alpha^{4}+\mathbf{C}_{3} \alpha^{3}+C_{2} \alpha^{2}+C_{1} \alpha+\mathbf{C}_{0},
$$

with

$$
\begin{aligned}
& \mathbf{C}_{4}=\mathbf{I}, \\
& \mathbf{C}_{3}=i \operatorname{Re} U \mathbf{I}, \\
& \mathbf{C}_{2}=-\left(i \omega \operatorname{Re} \mathbf{I}+2 \mathbf{D}^{2}\right), \\
& \mathbf{C}_{1}=i \operatorname{Re} U^{\prime \prime} \mathbf{I}-i \operatorname{Re} U \mathbf{D}^{2}, \\
& \mathbf{C}_{0}=\mathbf{D}^{4}+i \operatorname{Re} \omega \mathbf{D}^{2} .
\end{aligned}
$$


In the above set of equations, all the bold faced letters represent $(N+1) \times(N+1)$ matrices; the last four rows of these matrices are modified for the boundary conditions. Note that the boundary conditions are independent of the wave number, and therefore, are imposed only in $\mathbf{C}_{0}$ and the corresponding rows of the remaining matrices are set to zero. This implemantation creates a singular $\mathbf{C}_{4}$ matrix resulting in infinite eigenvalues. In order to remedy the infinite eigenvalue problem and to increase the accuracy by decreasing the number of arithmetic operations, the order of the matrices must be reduced. Since the zeros in the last four rows of the matrices (except $\mathbf{C}_{0}$ ) are to be preserved, the order reduction is done by simple column operations. This results in a $4 \times 4$ upper triangular submatrix in the last four rows of the $\mathbf{C}_{0}$ matrix (Fig. 1). If the boundary conditions are linearly independent, the order of the matrices is clearly reduced to $(N-3) \times(N-3)$ when four boundary conditions are eliminated. Here, note that each column switching necessitates the switching of the corresponding rows of the calculated eigenvector. Therefore, the original indices of the switched columns should be retained to decode the rows of the eigenvector once it is calculated.

Following Bridges and Morris ${ }^{6}$, and recalling that the eigenvalues of the companion matrix are the roots of the corresponding polynomial equation, a companion matrix for equation (15) can be written as

$$
\left\{\left(\begin{array}{cccc}
-\mathbf{C}_{3} & -\mathbf{C}_{2} & -\mathbf{C}_{1} & -\mathbf{C}_{0} \\
\mathbf{I} & \mathbf{0} & \mathbf{0} & \mathbf{0} \\
\mathbf{0} & \mathbf{I} & \mathbf{0} & \mathbf{0} \\
\mathbf{0} & \mathbf{0} & \mathbf{I} & \mathbf{0}
\end{array}\right)-\alpha\left(\begin{array}{cccc}
\mathbf{C}_{4} & \mathbf{0} & \mathbf{0} & \mathbf{0} \\
\mathbf{0} & \mathbf{I} & \mathbf{0} & \mathbf{0} \\
\mathbf{0} & \mathbf{0} & \mathbf{I} & \mathbf{0} \\
\mathbf{0} & \mathbf{0} & \mathbf{0} & \mathrm{I}
\end{array}\right)\right\}\left\{\begin{array}{c}
\alpha^{3} \phi \\
\alpha^{2} \phi \\
\alpha \phi \\
\phi
\end{array}\right\}=0 .
$$

This equation represents a complex generalized eigenvalue problem and can be solved by the QZ algoritm. Note that the order of the above system is four times larger than the original reduced problem. According to equation (17), the physical eigenfunctions can be directly obtained from the last quarter of the companion matrix equation (equation (16)). The numerical calculations were performed on the VAX/VMS 8550 at the University of Colorado at Boulder and the CYBER 205 at NASA Langley Research Center. This was accomplished by the use of three IMSL subroutines, GVLCG, GVCCG, CXLZ. 


\section{RESULTS, DISCUSSION AND CONCLUSION}

In this section, a comparison of our results to those of Bridges and Morris ${ }^{6}$ and Jordinson $^{7}$ is presented for the stability of channel and Blausius boundary layer flows, respectively.

a) Channel Flow Stability: Here, we concantrate on a test case given in Bridges and Morris $^{5}$ and also studied by Khorrami et al..$^{3}$.

For this purpose, we set $R e=6000$ and $\omega=0.26$ which is linearly unstable for this flow. The first seven members of the eigenvalue spectrum are tabulated and compared with those of Bridges and Morris ${ }^{6}$ in Table 1. Here increasing the order of Chebyshev polynomials results in noticable improvement in accuracy. However, machine and truncation errors impose a limit for the order of the polynomials that can be used; e. g. on the VAX/VMS 8550, increasing the order above 100 detoriates the eigenvalue spectrum. This behaviour suggests that a trade-off exists between the order of the Chebyshev polynomials and the ability of the QZ algorithm to accurately solve large matrices.

b) Blausius Boundary Layer Stability: In this section, following Jordinson ${ }^{7}$, we perform three calculations corresponding to subcritical $(R e=336, \omega=0.1297$ ), slightly unstable $(R e=598, \omega=0.1201)$ and unstable $(R e=998, \omega=0.1122)$ cases.

The computed least-damped eigenvalues are given in Table 2, and they are in excellent agreement with those of Jordinson ${ }^{7}$. Furthermore, the eigenfunctions and their derivatives are plotted in Figs. (2-4). Note that increasing viscosity, i. e. decreasing $R e$, pushes the peak of the derivative distribution toward the plate.

Another quantity of major interest is the Reynolds stress $(S)$ distribution across the domain which is given by ${ }^{7}$

$$
S=\alpha_{r}\left(\phi_{r} \phi_{i}^{\prime}-\phi_{r}^{\prime} \phi_{i}\right)-\alpha_{i}\left(\phi_{r} \phi_{r}^{\prime}+\phi_{i} \phi_{i}^{\prime}\right)
$$

where $r$ and $i$ refer to the real and imaginary parts of the eigenfunction or eigenvalue and the primes indicate differentiation with respect to $\mathrm{y}$. Recall that the Reynolds stress 
interacts with the mean velocity gradient to increase or decrease the energy of perturbations. The distributions of Reynolds stresses for the cases under consideration are presented in Fig. 5. As the damping decreases Reynolds stress remains positive over a larger range showing increasing energy transfer from the mean flow to the disturbances. Note also that the peaks are concentrated near the critical layer.

The results presented in this paper demonstrate the applicability of the Chebyshev matrix collocation method to nonlinear eigenvalue problems in semi-infinite domains using the companion matrix approach. The advantage of matrix collocation method in comparison with the tau method is the flexibility to use different coordinate transformations and to impose different boundary conditions with relative ease.

\section{REFERENCES}

1. S. A. Orszag, "Accurate Solution of the Orr-Sommerfeld Stability Equation" J. Fluid Mech. 50, 689-703 (1971).

2. C. Canuto, M. Y. Hussaini, A. Quarteroni, T. A. Zang, "Spectral Methods in Fluid Dynamics", Springer-Verlag (1988).

3. M. R. Khorrami, M. R. Malik, R. L. Ash, "Application of Spectral Collocation Techniques to the Stability of Swirling Flows" J. Comp. Phys. 81, 206-229 (1989).

4. E. Laurien, "Lösung der Orr-Sommerfeld-Gleichung für die Blasius'sche Grenzschichtströmung Mittels Chebyshev-Kollokation” DFVLR Report, IB 221-85 A 02 (1985).

5. S. Biringen, G. Danabasoglu, "Solution of the Orr-Sommerfeld Equation for the Blausius Boundary-Layer Documentation of Program ORRBL and a Test Case" NASA Contractor Report 4169 (1988).

6. T. J. Bridges, P. J. Morris, "Differential Eigenvalue Problems in Which the Parameter Appears Nonlinearly" J. Comp. Phys. 55, 437-460 (1984).

7. R. Jordinson, "The Flat Plate Boundary Layer. Part 1. Numerical Integration of the Orr-Sommerfeld Equation" J. Fluid Mech. 43, 801-811 (1970). 


\section{FIGURE CAPTIONS}

1. Structure of $\mathbf{C}_{\mathbf{0}}$ after simple column operations.

2. (a) Eigenfunction and (b) Derivative of eigenfunction for $R e=336$ and $\omega=0.1297$; real (A) and imaginary (B) parts (Blausius Boundary Layer).

3. (a) Eigenfunction and (b) Derivative of eigenfunction for $R e=598$ and $\omega=0.1201$; real (A) and imaginary (B) parts (Blausius Boundary Layer).

4. (a) Eigenfunction and (b) Derivative of eigenfunction for $R e=998$ and $\omega=0.1122$; real (A) and imaginary (B) parts (Blausius Boundary Layer).

5. Distribution of Reynolds stress; (A) $R e=336, \omega=0.1297$, (B) $R e=598, \omega=0.1201$ and (C) $R e=998, \omega=0.1122$.

Table 1: Comparison of the Eigenvalue Spectrum for Spatial Stability of Plane Poiseuille Flow - Without Reduction (Re $=6000, \omega=0.26$ )

\begin{tabular}{cccc}
\hline & Bridges and Morris & \multicolumn{2}{c}{ Present Method } \\
\hline Mode & & $N+1=41$ & $N+1=51$ \\
1 & $1.00047-\mathrm{i} 0.00086$ & $1.00046-\mathrm{i} 0.00086$ & $1.00047-\mathrm{i} 0.00086$ \\
2 & $0.28323+\mathrm{i} 0.02538$ & $0.28323+\mathrm{i} 0.02538$ & $0.28323+\mathrm{i} 0.02538$ \\
3 & $0.30165+\mathrm{i} 0.04886$ & $0.30165+\mathrm{i} 0.04886$ & $0.30165+\mathrm{i} 0.04886$ \\
4 & $0.31976+\mathrm{i} 0.07532$ & $0.31976+\mathrm{j} 0.07532$ & $0.31976+\mathrm{i} 0.07532$ \\
5 & $0.33745+\mathrm{i} 0.10492$ & $0.33748+\mathrm{i} 0.10485$ & $0.33745+\mathrm{i} 0.10492$ \\
6 & $0.35456+\mathrm{i} 0.13782$ & $0.35664+\mathrm{i} 0.13489$ & $0.35456+\mathrm{i} 0.13782$ \\
7 & $0.37090+\mathrm{i} 0.17425$ & & $0.37089+\mathrm{i} 0.17426$ \\
\hline
\end{tabular}

Table 2: Comparison of the Least Damped Eigenvalue for Spatial Stability of Blausius Boundary Layer

\begin{tabular}{cccc}
\hline Re & $\omega$ & Jordinson & Present Method \\
\hline 336 & 0.1297 & $0.3084+\mathrm{j} 0.0079$ & $0.30864+\mathrm{j} 0.00799$ \\
598 & 0.1201 & $0.3079-\mathrm{i} 0.0019$ & $0.30801-\mathrm{i} 0.00184$ \\
998 & 0.1122 & $0.3086-\mathrm{i} 0.0057$ & $0.30870-\mathrm{i} 0.00564$ \\
\hline
\end{tabular}




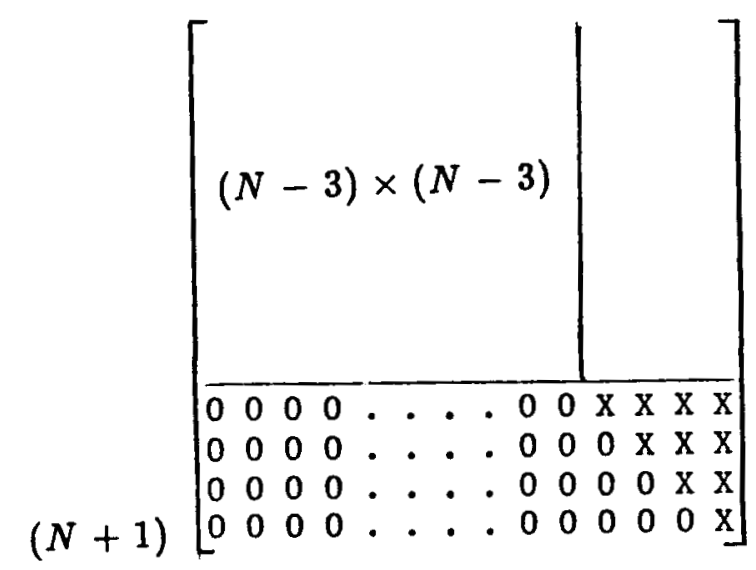

FIGURE 1

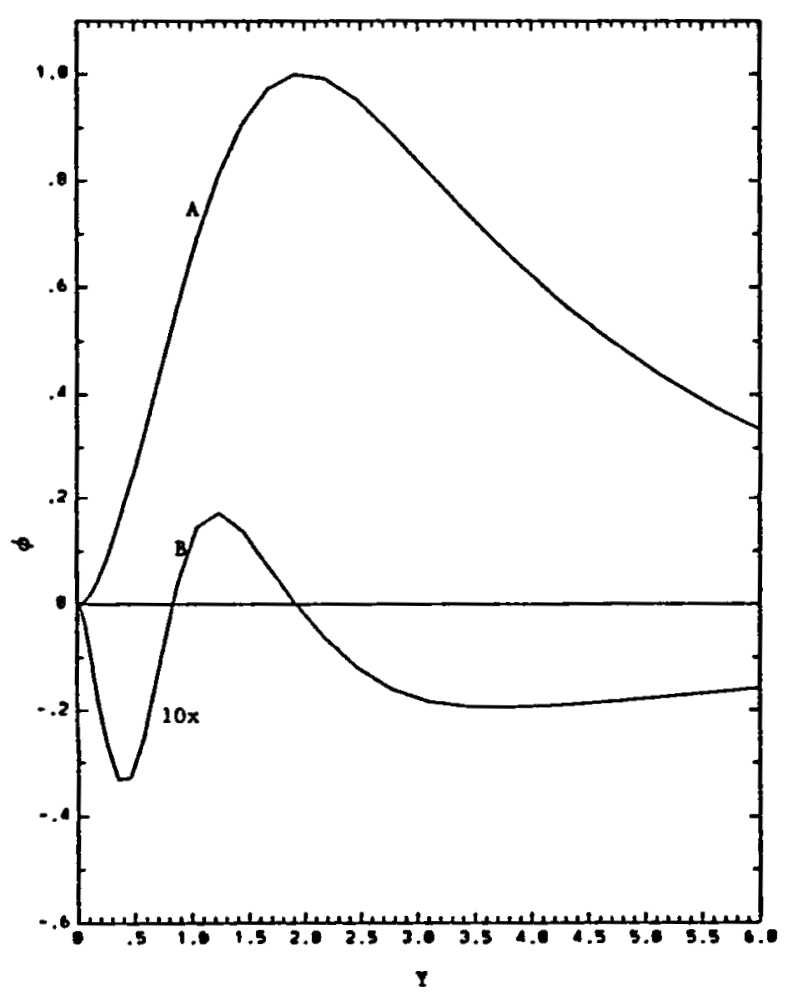

(a)

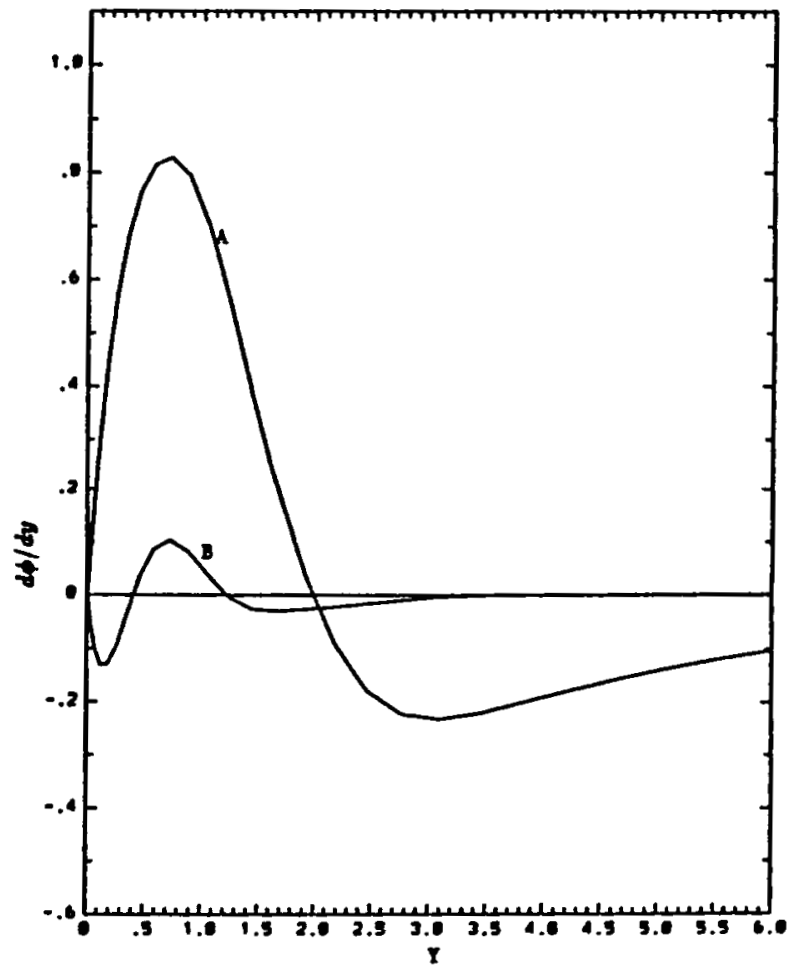

(b)

FIGURE 2 


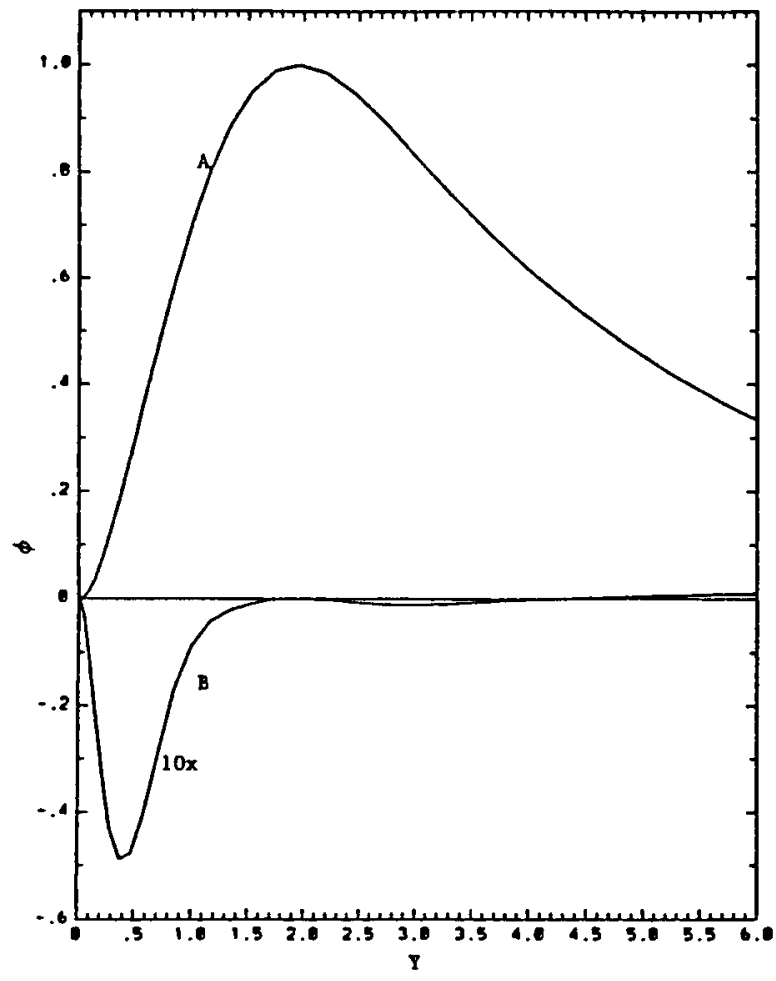

(a)

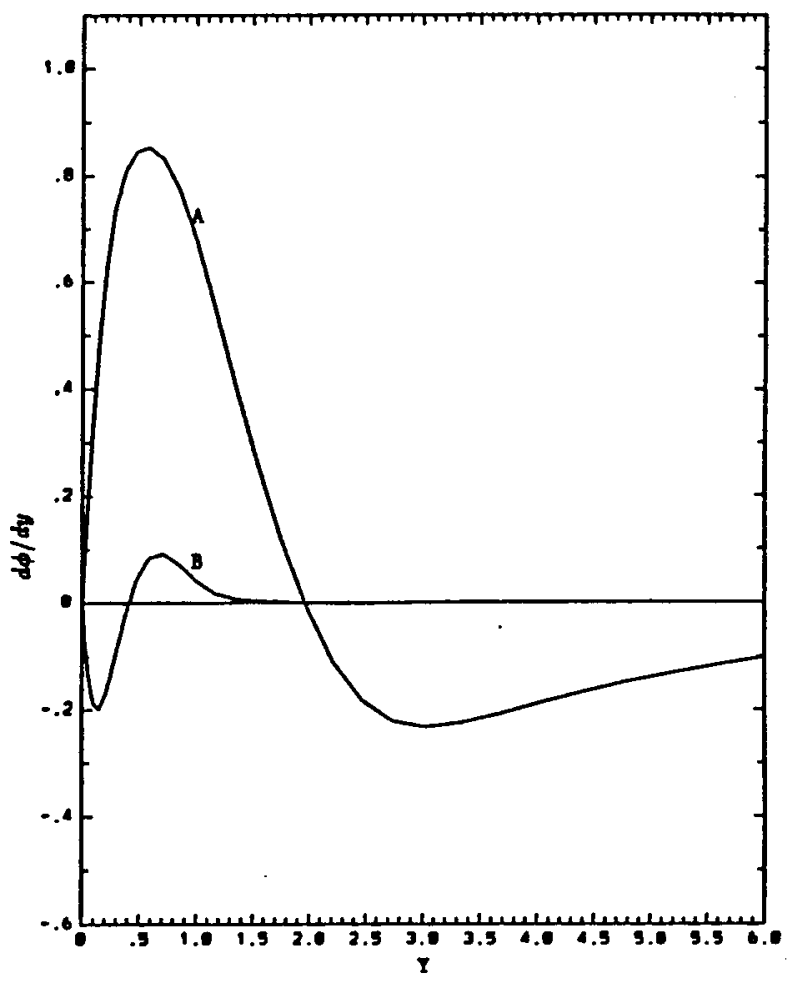

(b)

FIGURE 3

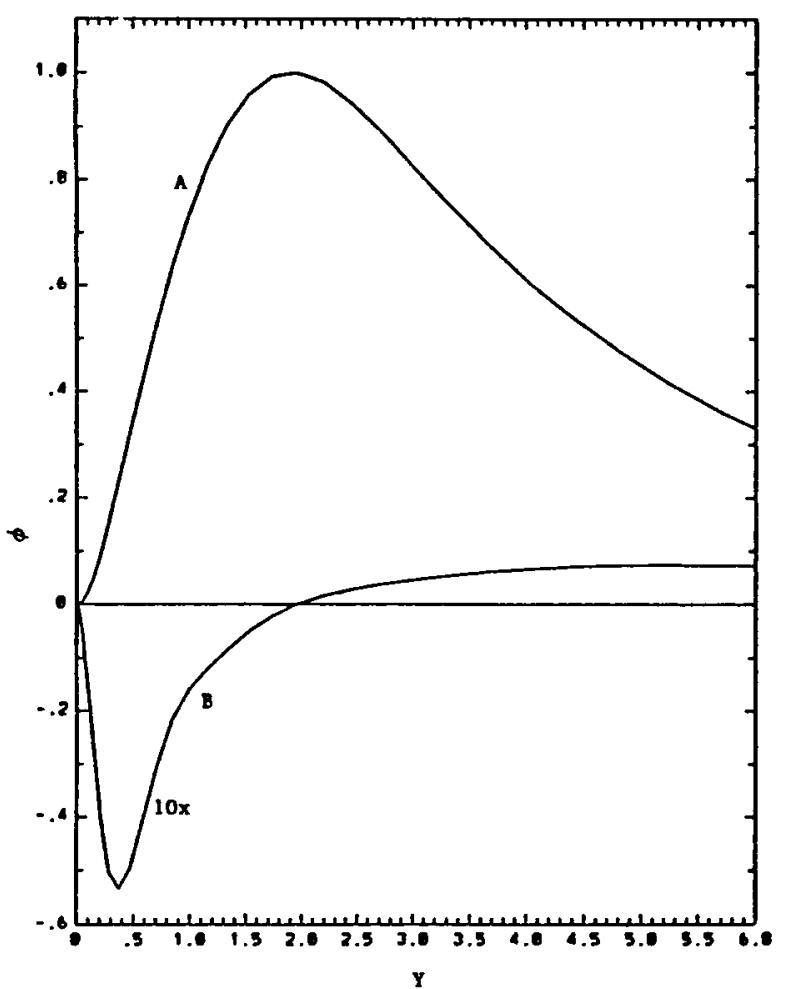

(a)

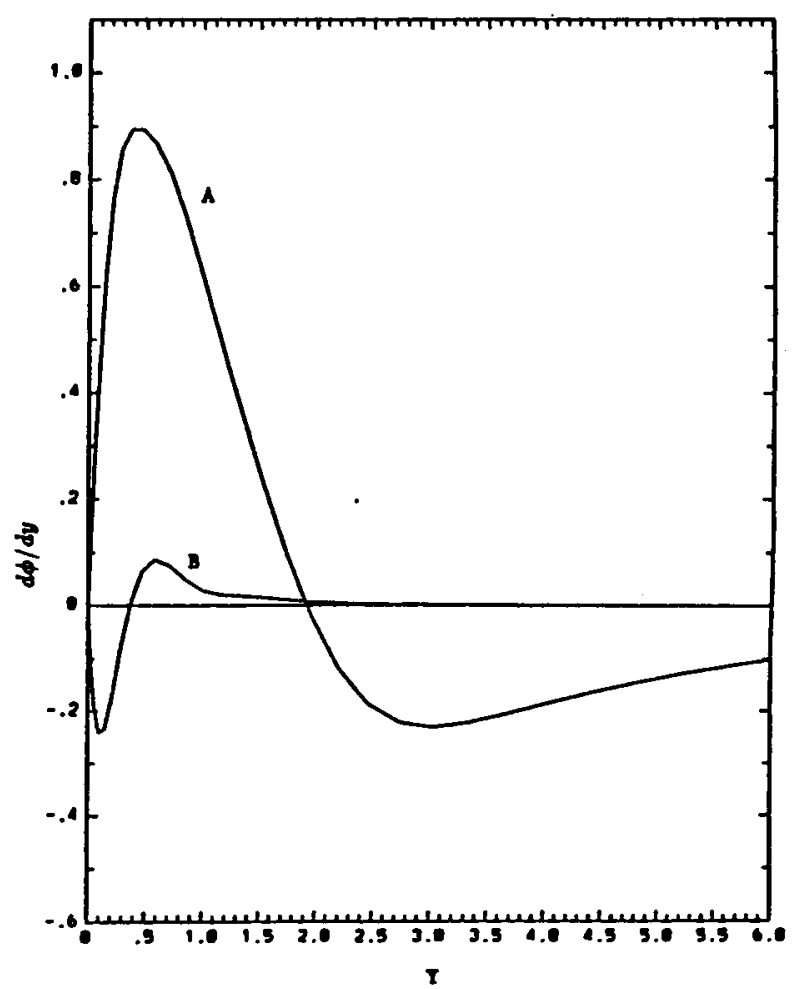

(b)

FIGUKE 4 


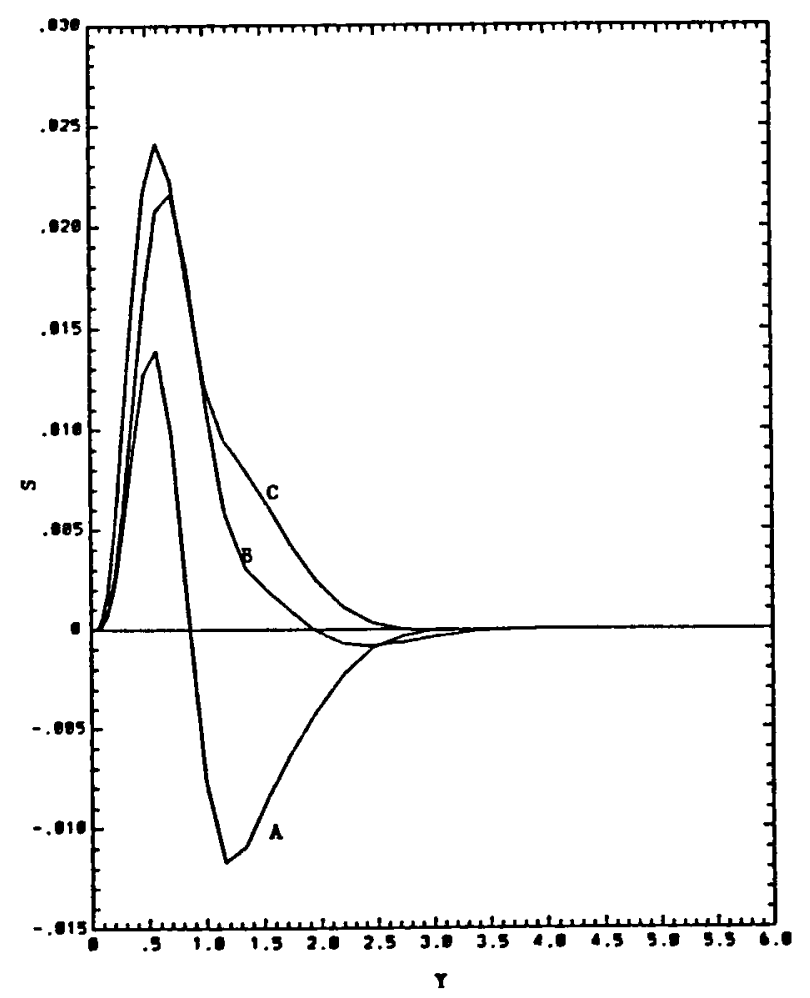

FIGURE 5 


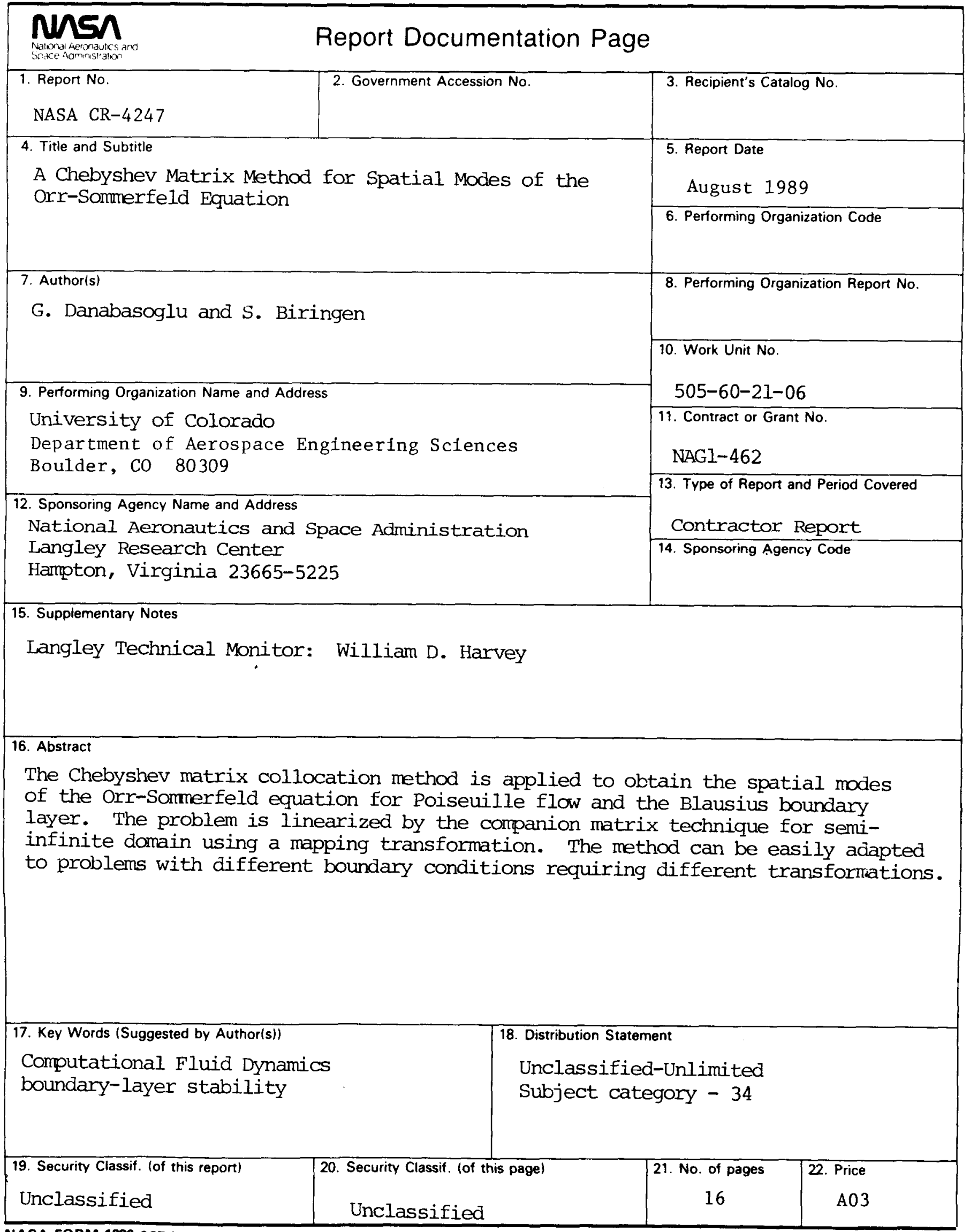

